\title{
BMJ Open Cardiac complications after stroke: protocol for a systematic review and meta-analysis
}

\author{
Guidelle Héloïse Kenmogne-Domning, ${ }^{1}$ Joseph Kamtchum-Tatuene, ${ }^{2,3}$ \\ Steve Raoul Noumegni, ${ }^{4}$ Christophe Maxime Fokoua-Dongmo, ${ }^{5}$ \\ Joseline Guetsop Zafack, ${ }^{6}$ Jean Jacques Noubiap ${ }^{7}$
}

To cite: KenmogneDomning GH, KamtchumTatuene J, Noumegni SR, et al. Cardiac complications after stroke: protocol for a systematic review and meta-analysis. BMJ Open 2018;8:e021416. doi:10.1136/ bmjopen-2017-021416

- Prepublication history and additional material for this paper are available online. To view these files, please visit the journal online (http://dx.doi. org/10.1136/bmjopen-2017021416).

GHK-D and JK-T contributed equally.

Received 28 December 2017 Revised 13 March 2018 Accepted 18 April 2018

Check for updates

For numbered affiliations see end of article.

Correspondence to Dr Joseph Kamtchum-Tatuene; jkamtchum@yahoo.fr

\section{ABSTRACT}

Introduction Stroke is the second most common cause of death after ischaemic heart diseases and the third leading cause of disability worldwide. The contribution of cardiac complications to the mortality of patients with stroke is variable across studies, ranging from $12.5 \%$ to $22.7 \%$. Many of these cardiac complications are preventable, and early recognition and adequate management guided by appropriate up-to-date knowledge of their relative incidence and fatality can help to improve patients' outcomes. This systematic review aims to summarise the available data on the burden of cardiac complications after stroke.

Methods and analysis This review will include all cross-sectional, case-control and cohort studies and clinical trials published between 1 January 1950 and 31 December 2017, involving adults and/or children, and reporting on the prevalence, the incidence and/or the mortality of cardiac complications after stroke. Two reviewers will independently screen titles and abstracts of records retrieved from PubMed, Excerpta Medica Database, ISI Web of Science and the Cumulative Index to Nursing and Allied Health Literature for eligibility, and then assess the risk of bias and quality of reporting to select the studies which will be included. All authors will contribute to the retrieval of full texts of eligible records and data extraction. Heterogeneity across studies will be evaluated by the $\chi^{2}$ test on Cochran's $Q$ statistic. Study-specific estimates of the prevalence, incidence and mortality of cardiac complications after stroke across studies will be pooled through random-effect or fixed-effect metaanalysis depending on the source of the heterogeneity, after stabilising the variance of individual studies using the Freeman-Tukey double arcsine transformation. Visual analysis of funnel plots and Egger's test will be done to detect small-study effect.

Ethics and dissemination This review and meta-analysis will be based on published data and will therefore not require a specific ethical clearance. The results will be published in peer-reviewed journals.

PROSPERO registration number CRD42018082551.

\section{INTRODUCTION}

Stroke is the second most common cause of death after ischaemic heart diseases and the third leading cause of disability worldwide. ${ }^{1-3}$

\section{Strengths and limitations of this study}

- This review will provide an up-to-date summary of the burden of cardiac complications after stroke, reflecting the changes in diagnosis and management of stroke and cardiac diseases over the past decades.

- We will use robust meta-analysis tools to provide reliable estimates of the prevalence, incidence and mortality of cardiac complications after acute stroke.

- One major limitation could be the predominance of data from randomised controlled trials which are known to recruit healthier patients, and this might underestimate the real-world incidence of cardiac complications after stroke.

Between 1990 and 2010, the burden of ischaemic and haemorrhagic stroke increased significantly in terms of the absolute number of people with incident ischaemic and haemorrhagic stroke $(37 \%$ and $47 \%$ increase, respectively), the number of deaths $(21 \%$ and $20 \%$ increase) and disability-adjusted life years (18\% and $14 \%$ increase) ${ }^{4}$ In 2015 , the number of stroke-related deaths was estimated to be 6.7 million. ${ }^{5}$ The initial neurological injury is responsible for the death in up to $43.9 \%$ of cases. ${ }^{6}$ The contribution of cardiac complications to the mortality of patients with stroke is variable across studies, ranging from $12.5 \%$ to $22.7 \% .^{6-8}$ Data from the Virtual International Stroke Trials Archive reveal that most serious cardiac complications occur within the first 14 days after stroke. ${ }^{6}$ They can arise as a direct consequence of the brain injury itself, from the ensuing reduction in mobility, or from stroke-related treatments. ${ }^{9}$ The spectrum of abnormalities includes hypertension, hypotension, myocardial infarction, repolarisation abnormalities, cardiac arrhythmias, left ventricular dysfunction and cardiac arrest. ${ }^{10} 11$ Many of these cardiac complications are preventable and 
when this is not possible, early recognition and treatment can improve patients' outcomes. ${ }^{9}$

In 2005, a systematic review of the risk of myocardial infarction and non-stroke vascular death after transient ischaemic attack and ischaemic stroke revealed an annual risk of $2.2 \%$ (1.7-2.7) for total myocardial infarction, $0.9 \%(0.7-1.2)$ for non-fatal myocardial infarction and $1.1 \%(0.8-1.5)$ for fatal myocardial infarction. ${ }^{12}$ However, the review did not consider other non-fatal cardiac complications after stroke. Furthermore, since the publication of this systematic review, the management of patients with stroke has changed dramatically to include earlier, longer and often more invasive cardiac monitoring, ${ }^{13} 14$ earlier and more aggressive treatment with thrombolysis and antithrombotics, ${ }^{15-17}$ increased availability of percutaneous coronarography intervention, which all might have changed the incidence and the mortality of cardiac complications after acute stroke. Here, we propose a protocol for a systematic review which aims at summarising the available data on the burden of cardiac complications after stroke.

\section{REVIEW QUESTION}

What is the burden of cardiac complications after stroke?

\section{OBJECTIVES}

To determine:

- The prevalence and the incidence of cardiac complications after stroke.

- The mortality rate of these complications.

\section{METHODS}

This review protocol has been prepared according to the 2015 Preferred Reporting Items for Systematic Reviews and Meta-Analyses Protocols guidelines (checklist provided as online supplementary appendix). ${ }^{18}$ The review is registered in the International Prospective Register for Systematic Reviews (registration number CRD42018082551).

\section{Criteria for considering studies for the review}

Inclusion criteria

We will include all cross-sectional, case-control and cohort studies and clinical trials published between 1 January 1950 and 31 December 2017, involving adults and/or children, and reporting on the prevalence, the incidence or the mortality of cardiac complications after stroke or providing enough data to compute these estimates. No language restriction will be applied.

\section{Exclusion criteria}

We will exclude reviews, commentaries, editorials, studies with small sample size (less than 30 participants), and studies lacking primary data or with incomplete methods description. For studies leading to more than one publication (duplicates), only the most comprehensive report including the largest sample size will be considered.

\section{Search strategy for the identification of relevant studies}

A comprehensive literature search will be performed in PubMed, Excerpta Medica Database (EMBASE), ISI Web of Science (Science Citation Index) and the Cumulative Index to Nursing and Allied Health Literature to identify potentially eligible studies. The literature search strategy is summarised in table 1 and table 2 for PubMed and EMBASE, respectively. Following the search in databases, we will screen the reference lists of eligible articles and relevant reviews as well as conference proceedings to identify additional sources of information. Search results will be compiled using the citation management software EndNote X6.0.1. The proposed start date for this review is 1 January 2018 and it is expected to be completed in a maximum of 6 months.

\section{Selection of studies for inclusion in the review}

Titles and abstracts of records identified through literature search will be independently screened for eligibility by two members of the research team (GHK-D and JK-T). Full texts of records deemed eligible will be retrieved and further assessed for inclusion by the same investigators. A screening guide will be developed to ensure consistency of the screening method applied by both assessors.

\section{Table 1 Search strategy for PubMed}

\section{Search terms}

\begin{tabular}{|c|c|}
\hline \#1 & $\begin{array}{l}\text { 'Stroke'[Tiab] OR ‘Transient ischemic attack'[Tiab] OR 'TIA'[Tiab] OR ‘Intracranial hemorrhage'[Tiab] OR 'Intracranial } \\
\text { haemorrhage'[Tiab] OR 'Subarachnoid hemorrhage'[Tiab] OR 'Subarachnoid haemorrhage'[Tiab] OR 'cerebrovascular } \\
\text { accident'[Tiab] }\end{array}$ \\
\hline \#2 & $\begin{array}{l}\text { 'Myocardial infarction'[Tiab] OR ‘acute coronary syndrome' [tiab] OR ‘myocardial ischaemia' [tiab] OR ‘Takotsubo' }[\text { Tiab] } \\
\text { OR ‘Wall motion abnormalities'[Tiab] OR ‘Neurogenic cardiac damage'[Tiab] OR 'Arrhythmia'[Tiab] OR ‘atrioventricular } \\
\text { block' [tiab] OR ‘sinoatrial block' [tiab] OR ‘atrial flutter' [tiab] OR ‘supraventricular tachycardia' [tiab] OR ‘ventricular } \\
\text { tachycardia' [tiab] OR ‘Atrial fibrillation'[Tiab] OR ‘QT prolongation' [tiab] OR 'torsade de pointes' [tiab] OR } \\
\text { 'hypertension' [tiab] OR ‘hypotension' [tiab] }\end{array}$ \\
\hline \#3 & ‘Mortality’[Tiab] OR ‘Death’[Tiab] OR ‘Fatality’[Tiab] OR ‘Prevalence'[Tiab] OR ‘Incidence'[Tiab] OR ‘Outcome’[Tiab] \\
\hline \#4 & \#1 AND \#2 AND \#3 \\
\hline \#5 & Restrict [humans] \\
\hline
\end{tabular}


Table 2 Search strategy for EMBASE

\section{Search tems}

\begin{tabular}{|c|c|}
\hline$\# 1$ & $\begin{array}{l}\text { ('cerebrovascular accident'/exp OR ‘cerebrovascular accident':ti,ab OR ‘stroke':ti,ab) OR ('brain } \\
\text { hemorrhage'/exp OR ‘brain hemorrhage':ti,ab OR ‘intracranial hemorrhage':ti,ab) }\end{array}$ \\
\hline \#2 & $\begin{array}{l}\text { 'hypertension': ti,ab OR 'hypotension': ti,ab OR 'heart infarction':ti,ab OR 'myocardial infarction':ti,ab } \\
\text { OR 'acute coronary syndrome':ti,ab OR 'heart muscle ischemia':ti,ab OR 'myocardial ischemia':ti,ab } \\
\text { OR 'takotsubo':ti,ab OR 'wall motion abnormalit':ti,ab OR 'neurogenic cardiac damage':ti,ab OR } \\
\text { 'atrioventricular block':ti,ab OR 'sinoatrial block':ti,ab OR 'atrial flutter':ti,ab OR 'supraventricular } \\
\text { tachycardia':ti,ab OR 'ventricular tachycardia':ti,ab OR 'atrial fibrillation':ti,ab OR 'qt prolongation':ti,ab }\end{array}$ \\
\hline$\# 4$ & \#1 AND \#2 AND \#3 \\
\hline \#6 (Restrict to humans) & \#5 AND 'human'/de \\
\hline $\begin{array}{l}\text { \#7 (Filter by study } \\
\text { type) }\end{array}$ & $\begin{array}{l}\text { \#6 AND ('clinical study'/de OR 'clinical trial'/de OR 'cohort analysis'/de OR 'comparative study'/de OR } \\
\text { 'controlled clinical trial'/de OR 'controlled study'/de OR 'family study'/de OR 'major clinical study'/de } \\
\text { OR 'medical record review'/de OR 'observational study'/de OR 'prospective study'/de OR 'randomized } \\
\text { controlled trial'/de OR 'retrospective study'/de OR 'systematic review'/de) }\end{array}$ \\
\hline
\end{tabular}

Any disagreement will be resolved by discussion and consensus. If the latter is not reached, arbitration will be sought from a third member of the team (JJN). The inter-rater agreement for the selection of studies will be assessed using a non-weighted Cohen's kappa statistic. ${ }^{19} 20$ Authors of publications reporting unclear data that may be subject to multiple interpretations will be contacted by email for clarification or to request supplemental information. If a study is excluded, the reasons will be documented.

\section{Assessment of the methodological quality and risk of bias}

The Risk of Bias Tool for Prevalence Studies ${ }^{21}$ and the Cochrane Risk of Bias Tool for randomised controlled trials ${ }^{22}$ will be used to evaluate the methodological quality and risk of bias for each study using the full-text publication. The quality of reporting of the studies included will be assessed using either the Strengthening the Reporting of Observational Studies in Epidemiology or the Consolidated Standards of Reporting Trials checklist depending on the nature of the study (observational study or clinical trial). ${ }^{2324}$ Risk of bias and quality of reporting scores will be presented in a table and inter-rater agreement will be assessed using a weighted Cohen's kappa statistic. ${ }^{25} 26$

\section{Data extraction and management}

A standardised data extraction sheet will be used to collect information on:

- Study identification: first author's name, year of publication, period of recruitment of participants, country.

- Study and participants' characteristics: study design (cross-sectional, cohort, case-control, clinical trial), setting (hospital-based, community-based), sample size, mean or median age, age range, proportion of male participants, proportion of patients with pre-existing cardiovascular risk factors (hypertension, diabetes mellitus, obesity, dyslipidaemia) or diseases (coronary artery disease, heart failure, previous stroke), mean or median stroke severity score on the
National Institute of Health Stroke Scale, proportion of patients with each type of cerebrovascular disease (ischaemic stroke, intracranial haemorrhage, subarachnoid haemorrhage), proportion of patients with lesion of the insula (if reported), proportion of patients with right hemisphere lesion (if reported), diagnostic criteria for stroke, proportion of patients who received intravenous thrombolysis, proportion of patients who received endovascular thrombectomy (without or with prior thrombolysis), mean (or median) time from stroke onset to the diagnosis of the reported cardiac complications (if available), duration of follow-up for cohort studies and clinical trials.

- Epidemiological estimates: prevalence, incidence and mortality of cardiac complications after stroke. Whenever these estimates are not readily available or computable using the primary data in the publication, the corresponding author will be contacted to request the missing information. The definition and diagnostic criteria used for each cardiac complication will also be reported. An illustrative list of the cardiac complications that will be considered in this review is provided in table 3 .

\section{Data analysis and reporting}

Data will be analysed using the metaprop command provided with the software STATA (V.13, StataCorp). ${ }^{27}$ Heterogeneity will be evaluated by the $\chi^{2}$ test on Cochran's Q statistic and quantified using $\mathrm{I}^{2}$ values, considering that $\mathrm{I}^{2}$ values of $25 \%, 50 \%$ and $75 \%$, represent low, medium and high heterogeneity, respectively. ${ }^{28}$ Subgroup analyses will be performed to detect possible sources of heterogeneity. Depending on whether the heterogeneity between effect estimates is most likely due to clinical or methodological diversity between studies, or due to random variation, study-specific estimates of the prevalence, incidence and mortality of cardiovascular 
Table 3 List of possible cardiac complications after stroke

$\begin{array}{ll}\begin{array}{ll}\text { Acute coronary } \\ \text { syndromes }\end{array} & \begin{array}{l}\text { Myocardial infarction } \\ \text { Regional wall motion abnormality } \\ \text { without infarction/neurogenic cardiac } \\ \text { damage/takotsubo cardiomyopathy }\end{array} \\ \begin{array}{ll}\text { Repolarisation } \\ \text { abnormalities }\end{array} & \begin{array}{l}\text { Abnormal waves and segments } \\ \text { ST-elevation }\end{array} \\ & \text { T waves (inversion or abnormal shape) } \\ & \text { U waves } \\ & \text { Q waves } \\ & \text { QT prolongation }\end{array}$

\begin{tabular}{ll} 
Arrhythmias & Atrial fibrillation \\
& Atrial flutter \\
& Supraventricular tachycardia \\
& Ventricular tachycardia \\
& Torsade de pointe \\
& Sinoatrial block \\
& Atrioventricular block \\
& Bundle block \\
Elevated cardiac & $\begin{array}{l}\text { Troponin } \\
\text { Creatine kinase-MB } \\
\text { Atrial natriuretic peptide } \\
\text { Others }\end{array}$ \\
& $\begin{array}{l}\text { Sudden cardiac death } \\
\text { Hypertension } \\
\text { Hypotension }\end{array}$ \\
\hline
\end{tabular}

complications after stroke across studies will be pooled through fixed-effect or random-effect meta-analysis, respectively, after stabilising the variance of individual studies using the Freeman-Tukey double arcsine transformation. ${ }^{29}{ }^{30}$ Study-specific estimates will be determined from the point estimate and the appropriate denominators, assuming a binominal distribution. Visual analysis of funnel plots and Egger's test will be done to detect smallstudy effect. ${ }^{31}$ All tests will be two-sided and statistical significance will be defined as $\mathrm{p}<0.05$.

The results of this systematic review will be reported according to the Meta-analysis Of Observational Studies in Epidemiology guidelines. ${ }^{32}$ The study selection process will be summarised using a flow diagram. Reasons for study exclusion will be described. Quantitative data will be presented in summary tables and forest plots where appropriate.

\section{Patient and Public Involvement statement}

No patients were involved in this study.

\section{Potential amendments}

We do not intend to make any amendment to this protocol. However, any necessary amendment will be documented and reported transparently.

\section{Ethics and dissemination}

This systematic review and meta-analysis will be based on published data and therefore will not require a specific ethical clearance. The results will be published in peer-reviewed journals and further presented at conferences. The review will be updated regularly as new data become available.

\section{Author affiliations}

${ }^{1}$ Higher Institute of Health Sciences, Universite des Montagnes, Bangangté, Cameroon

${ }^{2}$ Malawi-Liverpool-Wellcome Trust Clinical Research Programme, University of Malawi College of Medicine, Blantyre, Malawi

${ }^{3}$ Brain Infections Group, Institute of Infection and Global Health, University of Liverpool, Liverpool, UK

${ }^{4}$ Department of Public Health, Paris-Sud Faculty of Medicine, University of ParisSaclay, Paris, France

${ }^{5}$ School of Public Health, University at Albany, State University of New York, Albany, New York, USA

${ }^{6}$ Department of Social and Preventive Medicine, Laval University, Quebec, Canada ${ }^{7}$ Department of Medicine, University of Cape Town and Groote Schuur Hospital, Cape Town, South Africa

Contributors GHK-D, JK-T and JJN conceived the study. GHK-D and JK-T drafted the manuscript. SRN, CMF-D, GHK-D, JK-T, JGZ and JJN revised the manuscript. All authors approved the final version. JK-T is the guarantor of the review.

Funding The authors have not declared a specific grant for this research from any funding agency in the public, commercial or not-for-profit sectors.

Competing interests None declared.

Patient consent Not required.

Provenance and peer review Not commissioned; externally peer reviewed.

Open Access This is an Open Access article distributed in accordance with the Creative Commons Attribution Non Commercial (CC BY-NC 4.0) license, which permits others to distribute, remix, adapt, build upon this work non-commercially, and license their derivative works on different terms, provided the original work is properly cited and the use is non-commercial. See: http://creativecommons.org/ licenses/by-nc/4.0/

(C) Article author(s) (or their employer(s) unless otherwise stated in the text of the article) 2018. All rights reserved. No commercial use is permitted unless otherwise expressly granted.

\section{REFERENCES}

1. GBD 2016 Causes of Death Collaborators. Global, regional, and national age-sex specific mortality for 264 causes of death, 19802016: a systematic analysis for the global burden of disease study 2016. Lancet 2017:390:1151-210.

2. GBD 2016 DALYs and HALE Collaborators. Global, regional, and national disability-adjusted life-years (DALYs) for 333 diseases and injuries and healthy life expectancy (HALE) for 195 countries and territories, 1990-2016: a systematic analysis for the Global Burden of Disease Study 2016. Lancet 2017;390:1260-344.

3. GBD 2016 Disease and Injury Incidence and Prevalence Collaborators. Global, regional, and national incidence, prevalence, and years lived with disability for 328 diseases and injuries for 195 countries, 1990-2016: a systematic analysis for the Global Burden of Disease Study 2016. Lancet 2017;390:1211-59.

4. Krishnamurthi RV, Feigin VL, Forouzanfar MH, et al. Global and regional burden of first-ever ischaemic and haemorrhagic stroke during 1990-2010: findings from the global burden of disease study 2010. Lancet Glob Health 2013;1:e259-81.

5. WHO. Top 10 causes of death - Fact sheet $N^{\circ} 310$ Geneva: WHO Press. 2017 http://www.who.int/entity/mediacentre/factsheets/fs310/ en/index.html\# (cited 04 July 2017).

6. Prosser J, MacGregor L, Lees KR, et al. Predictors of early cardiac morbidity and mortality after ischemic stroke. Stroke 2007;38:2295-302.

7. Brønnum-Hansen H, Davidsen M, Thorvaldsen P. Long-term survival and causes of death after stroke. Stroke 2001;32:2131-6. 
8. Machado MF, Brucki SM, Nogueira CF, et al. Infectious disease is the most common cause of death among stroke patients: two-years of follow-up. Arq Neuropsiquiatr 2013;71:371-5.

9. Kumar S, Selim MH, Caplan LR. Medical complications after stroke. Lancet Neurol 2010;9:105-18.

10. Gregory T, Smith M. Cardiovascular complications of brain injury. Contin Educ Anaesth Crit Care Pain 2012;12:67-71.

11. Chen Z, Venkat $P$, Seyfried D, et al. Brain-heart interaction: cardiac complications after stroke. Circ Res 2017;121:451-68.

12. Touzé E, Varenne O, Chatellier G, et al. Risk of myocardial infarction and vascular death after transient ischemic attack and ischemic stroke: a systematic review and meta-analysis. Stroke 2005;36:2748-55.

13. Uphaus T, Grings A, Gröschel S, et al. Automatic detection of paroxysmal atrial fibrillation in patients with ischaemic stroke: better than routine diagnostic workup? Eur J Neurol 2017;24:990-4.

14. Carrazco C, Golyan D, Kahen M, et al. Prevalence and risk factors for paroxysmal atrial fibrillation and flutter detection after cryptogenic ischemic stroke. J Stroke Cerebrovasc Dis 2018;27.

15. Balami JS, Hadley G, Sutherland BA, et al. The exact science of stroke thrombolysis and the quiet art of patient selection. Brain 2013;136(Pt 12):3528-53.

16. Hacke W, Kaste M, Bluhmki E, et al. Thrombolysis with alteplase 3 to 4.5 hours after acute ischemic stroke. $N$ Engl J Med 2008;359:1317-29.

17. Berkhemer OA, Fransen PS, Beumer D, et al. A randomized trial of intraarterial treatment for acute ischemic stroke. $N$ Engl J Med 2015;372:11-20.

18. Moher $D$, Shamseer L, Clarke M, et al. Preferred reporting items for systematic review and meta-analysis protocols (PRISMA-P) 2015 statement. Syst Rev 2015;4:1.

19. Cohen J. A coefficient of agreement for nominal scales. Educ Psychol Meas 1960;20:37-46.

20. Sim J, Wright CC. The kappa statistic in reliability studies: use, interpretation, and sample size requirements. Phys Ther 2005;85:257-68.
21. Hoy D, Brooks $P$, Woolf $A$, et al. Assessing risk of bias in prevalence studies: modification of an existing tool and evidence of interrater agreement. J Clin Epidemiol 2012;65:934-9.

22. Higgins JP, Altman DG, Gøtzsche PC, et al. The cochrane collaboration's tool for assessing risk of bias in randomised trials. BMJ 2011;343:d5928.

23. Schulz KF, Altman DG, Moher D. CONSORT 2010 statement: updated guidelines for reporting parallel group randomised trials. $J$ Clin Epidemiol 2010;63:834-40.

24. von Elm E, Altman DG, Egger M, et al. The strengthening the reporting of observational studies in epidemiology (STROBE) statement: guidelines for reporting observational studies. Lancet 2007;370:1453-7.

25. Cohen J. Weighted kappa: nominal scale agreement with provision for scaled disagreement or partial credit. Psychol Bull 1968;70:213-20.

26. Viera AJ, Garrett JM. Understanding interobserver agreement: the kappa statistic. Fam Med 2005;37:360-3.

27. Nyaga VN, Arbyn M, Aerts M. Metaprop: a Stata command to perform meta-analysis of binomial data. Arch Public Health 2014;72:39.

28. Huedo-Medina TB, Sánchez-Meca J, Marín-Martínez F, et al. Assessing heterogeneity in meta-analysis: Q statistic or 12 index? Psychol Methods 2006;11:193-206.

29. Barendregt JJ, Doi SA, Lee YY, et al. Meta-analysis of prevalence. J Epidemiol Community Health 2013;67:974-8.

30. Freeman MF, Tukey JW. Transformations related to the angular and the square root. Ann Math Statist 1950;21:607-11.

31. Sterne JA, Egger M, Moher D. Addressing reporting biases. In: Higgins JP, Green S, eds. Cochrane handbook for systematic reviews of interventions. Chichester: Wiley, 2008:297-334.

32. Stroup DF, Berlin JA, Morton SC, et al. Meta-analysis of observational studies in epidemiology: a proposal for reporting. Meta-analysis of observational studies in epidemiology (MOOSE) group. JAMA 2000;283:2008-12. 